\title{
8-羟基喹啉席夫碱衍生物及其金属配合物的合成及苂光性质研究
}

\author{
王辉* 张永红陶敬奇曾卓曾和平 \\ (华南师范大学化学与环境学院 广东广州 510006)
}

\begin{abstract}
摘要 设计合成了三种新的 8-羟基喹啉席夫碱衍生物 4-(8-羊基喹啉-5-亚胺甲基)-7-甲氧基苯并吡喃-2-酮(3a)，4-(8-羟 基喹啉-5-亚胺甲基)-7-已氧基苯并吡喃-2-酮(3b)和 4-(8-差基喹啉-5-亚胺甲基)-7-十八烷氧基苯并吡喃-2-酮(3c)及其铝、 锌配合物, 产物结构经 ${ }^{1} \mathrm{H}\left({ }^{13} \mathrm{C}\right) \mathrm{NMR}, \mathrm{MS}, \mathrm{HRMS}, \mathrm{IR}$ 和元素分析表征, 研究了它们的苂光发光性能.

关键词 8-着基喹啉衍生物; 席夫碱; 金属配合物; 菼光性质
\end{abstract}

\section{Synthesis and Photoluminescence Properties of 8-Hydroxyquinoline Schiff Base Derivatives and Their Metal Complexes}

\author{
Wang, Hui* Zhang, Yonghong Tao, Jingqi Zeng, Zhuo Zeng Heping \\ (School of Chemistry and Environment, South China Normal University, Guangzhou 510006)
}

\begin{abstract}
Three new 8-hydroxyquinoline Schiff base derivatives, such as 4-[(8-hydroxyquinolin-5-ylimino)methyl]7-methoxy-2H-chromen-2-one (3a), 4-[(8-hydroxyquinolin-5-ylimino)methyl]-7-hexyloxy-2H-chromen-2-one (3b), 4-[(8-hydroxyquinolin-5-ylimino)methyl]-7-octadecyloxy-2H-chromen-2-one (3c), and their aluminium and zinc complexes have been synthesized. Compound structures were identified by means of ${ }^{1} \mathrm{H}\left({ }^{13} \mathrm{C}\right) \mathrm{NMR}$, IR, MS, HRMS and elemental analysis. Their fluorescence properties were discussed.
\end{abstract}

Keywords 8-hydroxyquinoline derivative; Schiff base; metal complex; fluorescence property

8 -羊基喹啉铝是一种介于无机物与有机物之间的 发光材料, 具有电子传输性能, 可用真空蒸镀法形成稳 定无瑕的薄膜，其作为发光层夹在阴极金属材料与具有 空穴传输性能的有机物之间组成复合发光材料, 可有效 缓解有机物与无机物之间的界面断裂现象，是一种综合 性能优秀的发光材料. 自从美国柯达公司研究人员 ${ }^{[1]}$ 首 次报道以二胺衍生物为空穴传输层, 8-羟基喹啉铝为发 光层的双层结构有机薄膜电致发光器件以来, 8-着基喹 啉铝成为小分子电致发光材料的研究热点 ${ }^{[2-8]}$.

Anzenbacher 等 ${ }^{[9,10]}$ 在 8-差基喹啉的 5-位上连接共 轭基团, 合成了系列 8-羊基喹啉衍生物并将其与 $\mathrm{Al}^{3+}$ 配 位形成配合物, 结果发现引入不同的共轭体系, 可以方 便地调节配合物发光波长, 调节范围为 $490 \sim 612 \mathrm{~nm}$; Burrows 等 ${ }^{[11]}$ 又发现选用不同的金属离子与 8 -差基喹啉 衍生物进行配位, 也可改变配合物的发光性质, 因此对 配体 8-羟基喹啉的结构进行修饰改造, 或者采用不同的 配位金属, 通过改变发光材料分子 HOMO 与 LUMO 间
的能隙差，可以达到调控发光波长的目的，获得不同发 光波长甚至发白光的发光材料. 香豆素是一种重要的反 应中间体，而其本身就具有良好的发射绿色荧光性能， 也是制作电致发光器件的材料之一 ${ }^{[12 \sim 14]}$. 为了寻找更 多的复合发光材料, 本课题组采用 5-氨基-8-羟基喹啉 与 4-甲酰基-7 烷氧基香豆素反应, 获得了三种 8-差基喹 啉的席夫碱衍生物配体, 并将其与金属铝和锌配位, 形 成了三种新的 8-手基喹啉席夫碱金属化合物, 并研究了 这些化合物的光物理性质. 合成路线见 Scheme 1.

金属配合物 $\mathrm{Al}(\mathbf{3 a})_{3} \sim \mathrm{Al}(\mathbf{3 c})_{3}$ 和 $\mathrm{Zn}(\mathbf{3 a})_{2} \sim \mathrm{Zn}(\mathbf{3 c})_{2}$ 结 构见 Scheme 2.

\section{1 实验部分}

\section{1 仪器与试剂}

美国 VARIAN INONA 400MHz 核磁共振仪, TMS 为内标, $\mathrm{DMSO}$ 或 $\mathrm{CDCl}_{3}$ 为溶剂; 日本 JASCO UV-530

*E-mail: huiwang@scnu.edu.cn

Received July 2, 2011; revised October 5, 2011; accepted October 25, 2011.

Project supported by the Science and Technology Plan Project of Guangdong Province (No. 2008B010600008).

广东省科技计划(No. 2008B010600008)资助项目。 


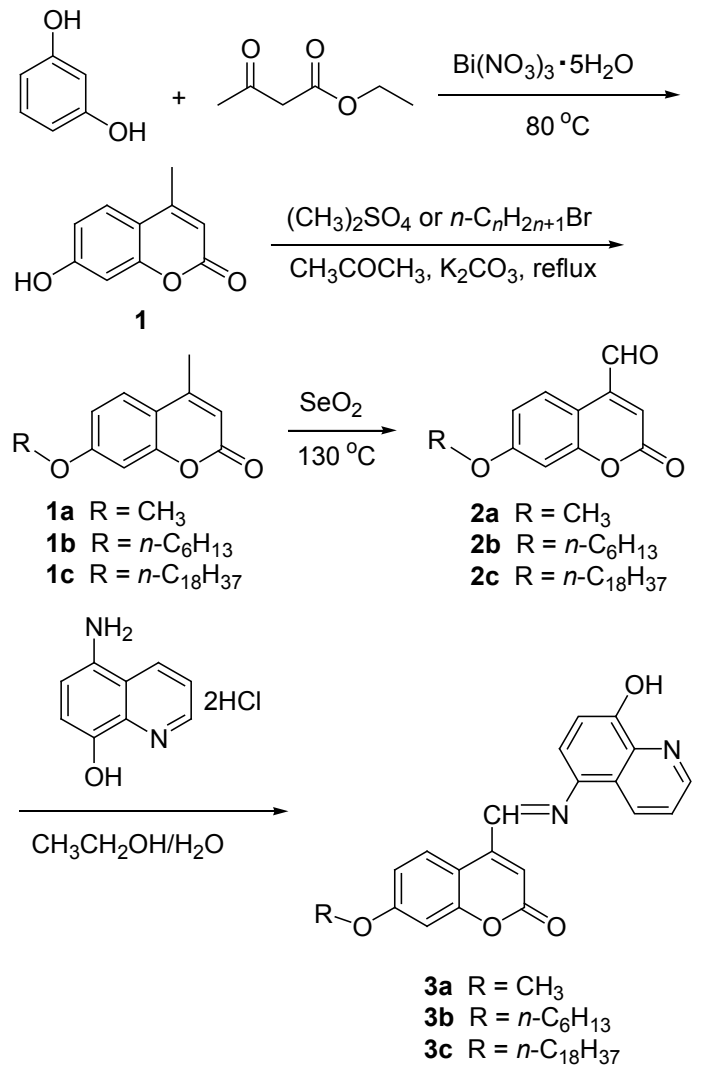

Scheme 1

紫外-可见分光光度计; 日本岛津 FT-IR 红外光谱仪; 美国 PERKIN- ELMER 1730 FT-IR 红外光谱仪; 美国 THEROMO LCQ DECA XP MAX 质谱仪; 德国 NETZSCH 公司 STA409PC 热重分析仪; 北京泰克双目 显微熔点测定仪. 5-氨基-8-羊基喹啉盐酸盐购自百灵威 公司, 柱层析硅胶为青岛海洋化工厂生产(200 300 目), 其它溶剂或试剂均为分析纯，未处理直接使用.

\section{2 化合物 4-甲基-7-羟基苯并吡喃-2-酮(1)的合成}

4-甲基-7-差基苯并吡喃-2-酩(1)的合成按文献[15] 的方法进行, 粗产物经无水乙醇重结晶后得到白色粉末 状固体, 产率 96\%, m.p. $183 \sim 185{ }^{\circ} \mathrm{C}$.

\section{3 化合物 $1 \mathrm{a} \sim 1 \mathrm{c}$ 的合成}

\subsection{1 化合物 $1 \mathrm{a}$ 的合成}

4-甲基-7-甲氧基苯并吡喃-2-酮(1a)的合成按照文 献[16]的方法进行, 粗产物经无水乙醇重结晶得到白色 固体, 产率 $84 \%$, m.p. $158 \sim 160{ }^{\circ} \mathrm{C}$.

\subsection{2 化合物 $1 \mathrm{~b}$ 和 $1 \mathrm{c}$ 的合成}

在盛有 $30 \mathrm{~mL}$ 丙酮的圆底烧瓶中加入 $1.134 \mathrm{~g}(6.4$ $\mathrm{mmol}$ ) 4-甲基-7-羊基苯并吡喃-2-酮、12.8 $\mathrm{mmol}$ 正澳己 烷 (1-澳十八碳烷)、13.3 $\mathrm{g}$ 无水 $\mathrm{K}_{2} \mathrm{CO}_{3}$ 和少量 $\mathrm{KI}$, 摚 拌回流 $12 \mathrm{~h}$. 反应液过滤, 滤液减压浓缩除去溶剂, 剩 余物再经柱层析纯化, 用石油醚-乙酸乙酯 $(V: V=4: 1$

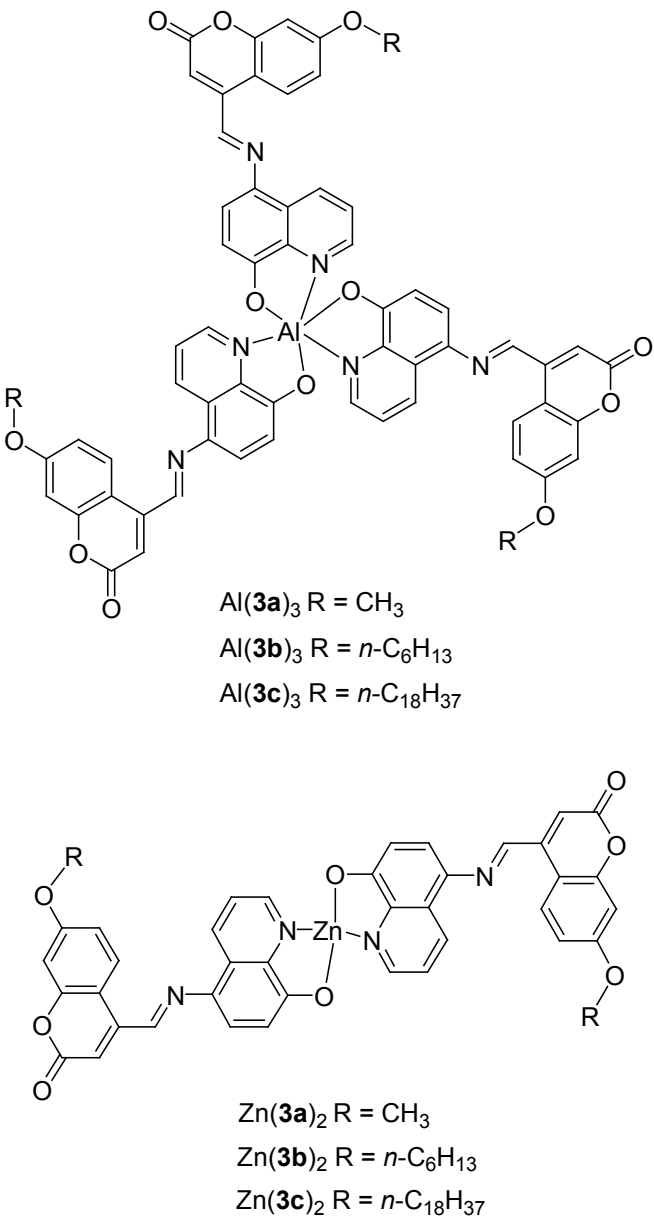

Scheme 2

或 5：1)作为洗脱剂, 得化合物 $\mathbf{1 b}$ 和 1c.

4-甲基-7-已氧基苯并吡喃-2-酮(1b): 淡黄色的液 体, 产率 94\%. ${ }^{1} \mathrm{H}$ NMR $\left(400 \mathrm{MHz}, \mathrm{CDCl}_{3}\right) \delta: 0.88(\mathrm{t}, J=$ $6.8 \mathrm{~Hz}, 3 \mathrm{H}), 1.31 \sim 1.43(\mathrm{~m}, 6 \mathrm{H}), 1.76 \sim 1.82(\mathrm{~m}, 2 \mathrm{H}), 2.35$ (s, 3H), 3.97 (t, $J=6.8 \mathrm{~Hz}, 2 \mathrm{H}), 6.08(\mathrm{~s}, 1 \mathrm{H}), 6.75(\mathrm{~s}, 1 \mathrm{H})$, $6.81(\mathrm{~d}, J=8.8 \mathrm{~Hz}, 1 \mathrm{H}), 7.45(\mathrm{~d}, J=8.8 \mathrm{~Hz}, 1 \mathrm{H}) ;{ }^{13} \mathrm{C} \mathrm{NMR}$ $\left(100 \mathrm{MHz}, \mathrm{CDCl}_{3}\right) \delta: 162.4,161.1,155.8,152.7,126.2$, 113.3, 112.7, 112.4, 101.5, 68.8, 31.6, 29.1, 26.0, 23.2, 19.3，14.2; FT-IR (KBr) v: 3023, 2927, $1733(\mathrm{C}=\mathrm{O})$, 1608, 1550, 1506, 1395, 1381, 1282, 1141, $1073 \mathrm{~cm}^{-1}$; ESI-MS $m / z$ (\%): $261.45\left([\mathrm{M}+\mathrm{H}]^{+}, 15\right)$.

4-甲基-7-十八碳烷氧基苯并吡喃-2-酮(1c): 淡黄色 固体, 产率 85\%. m.p. $38 \sim 40{ }^{\circ} \mathrm{C} ;{ }^{1} \mathrm{H}$ NMR $(400 \mathrm{MHz}$, $\left.\mathrm{CDCl}_{3}\right) \delta: 0.80(\mathrm{t}, J=6.8 \mathrm{~Hz}, 3 \mathrm{H}), 1.18 \sim 1.50(\mathrm{~m}, 20 \mathrm{H})$, $1.70 \sim 1.81(\mathrm{~m}, 12 \mathrm{H}), 2.31(\mathrm{~s}, 3 \mathrm{H}), 3.93(\mathrm{t}, J=6.8 \mathrm{~Hz}, 2 \mathrm{H})$, $6.04(\mathrm{~s}, 1 \mathrm{H}), 6.72(\mathrm{~d}, J=2.4 \mathrm{~Hz}, 1 \mathrm{H}), 6.77$ (dd, $J=2.4,8.8$ $\mathrm{Hz}, 1 \mathrm{H}), 7.40(\mathrm{~d}, J=8.8 \mathrm{~Hz}, 1 \mathrm{H}) ;{ }^{13} \mathrm{C}$ NMR $(100 \mathrm{MHz}$, $\left.\mathrm{CDCl}_{3}\right) \delta: 162.3,161.3,155.3,152.5,125.4,113.4,112.7$, $111.8,101.4,68.6,33.9,33.6,32.9,31.9,30.5,29.7$ (2C), 29.6 (2C), 29.5, 29.4, 28.8, 28.6, 28.2, 26.0, 22.7, 18.6, 
14.1; FT-IR (KBr) v: 2929, 2846, $1720(\mathrm{C}=\mathrm{O}), 1616$, 1509, 1462, 1385, 1289, 1144, 1070, $1015 \mathrm{~cm}^{-1}$; ESI-MS $m / z(\%): 879.51\left([2 \mathrm{M}+\mathrm{Na}]^{+}, 100\right) ; 429.71\left([\mathrm{M}+\mathrm{H}]^{+}, 5\right)$.

\section{4 化合物 $2 a \sim 2 c$ 的合成}

在盛有 $25 \mathrm{~mL}$ 二甲苯的圆底烧瓶中加入 $5.3 \mathrm{mmol}$ 4-甲基-7-甲氧基苯并吡喃-2-酮(4-甲基-7-己氧基苯并吡 喃-2-酮、4-甲基-7-十八碳烷氧基苯并吡喃-2-酮), 搅拌 溶解后, 再加入 $1.179 \mathrm{~g}$ (10.6 mmol) $\mathrm{SeO}_{2}, 130{ }^{\circ} \mathrm{C}$ 下反应 $20 \mathrm{~h}$, 趁热过滤, 滤液冷却, 析出黄色固体, 再次过滤, 固体用石油醚洗涤，得到化合物 $\mathbf{2 a} \sim 2 \mathbf{c}$ ，产率 $67 \%$ $83 \%$.

4-甲酰基-7-甲氧基苯并吡喃-2-酮(2a): 淡黄色固 体, 产率 70\%. m.p. $188 \sim 190{ }^{\circ} \mathrm{C} ;{ }^{1} \mathrm{H}$ NMR $(400 \mathrm{MHz}$, $\left.\mathrm{CDCl}_{3}\right) \delta: 10.06(\mathrm{~s}, 1 \mathrm{H}), 8.48(\mathrm{~d}, J=9.2 \mathrm{~Hz}, 1 \mathrm{H}), 6.91(\mathrm{dd}$, $J=2.4,8.8 \mathrm{~Hz}, 1 \mathrm{H}), 6.85(\mathrm{~d}, J=2.4 \mathrm{~Hz}, 1 \mathrm{H}), 6.70(\mathrm{~s}, 1 \mathrm{H})$, 3.88 (s, 3H); ${ }^{13} \mathrm{C}$ NMR (100 MHz, $\left.\mathrm{CDCl}_{3}\right) \delta: 191.8,162.8$, 160.7 , 156.4, 143.7, 127.3, 121.8, 113.6, 107.8, 101.5, 55.7; FT-IR (KBr) v: 3021, 2974, 2857, 1725 (C=O), 1655, 1554, 1506, 1458, 1212, 1185, $1036 \mathrm{~cm}^{-1}$; ESI-MS $m / z(\%): 205\left([\mathrm{M}+\mathrm{H}]^{+}, 45\right)$.

4-甲酰基-7-已氧基苯并吡喃-2-酮(2b): 黄色固体, 产率 67\%. m.p. $148 \sim 151{ }^{\circ} \mathrm{C} ;{ }^{1} \mathrm{H}$ NMR (400 MHz, $\left.\mathrm{CDCl}_{3}\right)$ $\delta$ : 10.05 (s, 1H, CHO), 8.45 (d, $J=9.2 \mathrm{~Hz}, 1 \mathrm{H}), 6.89$ (dd, $J=9.2,1.2 \mathrm{~Hz}, 1 \mathrm{H}), 6.82(\mathrm{~d}, J=1.2 \mathrm{~Hz}, 1 \mathrm{H}), 6.68(\mathrm{~s}, 1 \mathrm{H})$, $4.01\left(\mathrm{t}, J=6.4 \mathrm{~Hz}, 2 \mathrm{H}, \mathrm{OCH}_{2}\right), 1.77 \sim 1.84(\mathrm{~m}, 2 \mathrm{H})$, $1.42 \sim 1.48(\mathrm{~m}, 2 \mathrm{H}), 1.32 \sim 1.35(\mathrm{~m}, 4 \mathrm{H}), 0.90(\mathrm{t}, J=5.6$ $\mathrm{Hz}, 3 \mathrm{H}) ;{ }^{13} \mathrm{C} \mathrm{NMR}\left(100 \mathrm{MHz}, \mathrm{CDCl}_{3}\right) \delta: 191.8,162.9$, $160.9,156.4,143.7,127.2,121.9,113.6,107.9,101.5$, 68.7, 31.5, 28.8, 25.6, 22.5, 14.0; FT-IR (KBr) v: 2934, 2857, $1728(\mathrm{C}=\mathrm{O}), 1613,1550,1508,1380,1292,1187$, 1149, $1056 \mathrm{~cm}^{-1}$; ESI-MS $m / z(\%): 275\left([\mathrm{M}+\mathrm{H}]^{+}, 5\right)$.

4-甲酰基-7-十八碳烷氧基苯并吡喃-2-酥(2c): 黄色 固体, 产率 $83 \%$. m.p. $86 \sim 88{ }^{\circ} \mathrm{C} ;{ }^{1} \mathrm{H}$ NMR $(400 \mathrm{MHz}$, $\left.\mathrm{CDCl}_{3}\right) \delta: 0.85(\mathrm{t}, J=6.8 \mathrm{~Hz}, 3 \mathrm{H}), 1.23 \sim 1.44(\mathrm{~m}, 32 \mathrm{H})$, $4.00(\mathrm{t}, J=6.8 \mathrm{~Hz}, 2 \mathrm{H}), 6.67(\mathrm{~s}, 1 \mathrm{H}), 6.82(\mathrm{~d}, J=2.4 \mathrm{~Hz}$, $1 \mathrm{H}), 6.88(\mathrm{dd}, J=2.4,8.8 \mathrm{~Hz}, 1 \mathrm{H}), 8.44$ (d, $J=8.8 \mathrm{~Hz}$, 1H), $10.04(\mathrm{~s}, 1 \mathrm{H}) ;{ }^{13} \mathrm{C} \mathrm{NMR}\left(100 \mathrm{MHz}, \mathrm{CDCl}_{3}\right) \delta: 191.8$, $162.9,160.8,156.5,143.7,127.2,121.9,113.6,107.9$, 101.5, 68.7, 31.9, 29.69 (6C), 29.66, 29.65, 29.57, 29.53, 29.36, 29.32, 28.9, 25.9, 22.7, 14.1; FT-IR (KBr) v: 3076, 2917, 2846, 1733 (C=O), 1610, 1473, 1390, 1338, 1264, 1149, $1059 \mathrm{~cm}^{-1}$; ESI-MS m/z (\%): $443\left([\mathrm{M}+\mathrm{H}]^{+}, 10\right)$.

\section{5 化合物 $3 a \sim 3 c$ 的合成}

在氮气氛围中, 将 $58 \mathrm{mg}(0.25 \mathrm{mmol}) 5$-氨基- 8 -差 基喹啉盐酸盐和 $3 \mathrm{~mL}$ 去离子水加入 $100 \mathrm{~mL}$ 三口烧瓶

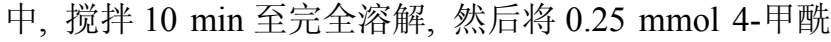
基-7-甲氧基苯并吡喃-2-酮(4-甲酰基-7-己氧基苯并吡 喃-2-酩、4-甲酰基-7-十八碳烷氧基苯并吡喃-2-酥)溶于 $10 \mathrm{~mL}$ 无水乙醇, 用恒压漏斗逐滴加入到反应烧瓶中, 约 $1 \mathrm{~h}$. 滴加完毕. 所得混合物室温摚拌反应 $3 \mathrm{~h}$, 有黄 色沉淀析出, 将反应液转移到试管中, 静置 $2 \mathrm{~h}$., 离心 分离出沉淀, 用少量无水乙醇洗涤沉淀 2 3 次, 真空干 燥得 3a 3c, 产率 $62 \% \sim 78 \%$.

4-(8-差基喹啉-5-亚氨基甲基)-7-甲氧基苯并吡喃2-酩(3a)：淡黄色固体，产率 $62 \%$. 熔点测定至 $245{ }^{\circ} \mathrm{C}$ 开 始分解; ${ }^{1} \mathrm{H}$ NMR (400 MHz, DMSO- $\left.d_{6}\right) \delta: 10.11(\mathrm{~s}, 1 \mathrm{H}$, $\mathrm{OH}), 9.13$ (s, 1H), 9.06 (d, $J=9.2 \mathrm{~Hz}, 1 \mathrm{H}), 9.03$ (d, $J=4.4$ $\mathrm{Hz}, 1 \mathrm{H}), 8.86$ (d, $J=9.6 \mathrm{~Hz}, 1 \mathrm{H}), 7.84(\mathrm{dd}, J=4.4,8.0 \mathrm{~Hz}$, 1H), 7.79 (d, $J=8.4 \mathrm{~Hz}, 1 \mathrm{H}), 7.32$ (d, $J=8.0 \mathrm{~Hz}, 1 \mathrm{H})$, $7.09 \sim 7.11(\mathrm{~m}, 2 \mathrm{H}), 6.99(\mathrm{~m}, 1 \mathrm{H}), 3.91(\mathrm{~s}, 3 \mathrm{H}) ;{ }^{13} \mathrm{C} \mathrm{NMR}$ $\left(100 \mathrm{MHz}, \mathrm{DMSO}-d_{6}\right) \delta: 167.79,164.15,162.90,160.87$, $156.31,156.24,148.43,147.00,146.22,137.58,128.23$, $126.25,122.98,116.49,115.30,112.96,110.68,104.99$, 101.61, 56.43; FT-IR (KBr) v: 3425, 2954, 2916, 1700 $(\mathrm{C}=\mathrm{O}), 1613(\mathrm{C}=\mathrm{N}), 1557,1520,1470,1413,1375$, 1348, 1208, 1135, $1019 \mathrm{~cm}^{-1}$; EI-MS m/z (\%): $346\left([\mathrm{M}]^{+}\right.$, 35); HRMS calcd for $\mathrm{C}_{20} \mathrm{H}_{14} \mathrm{~N}_{2} \mathrm{O}_{4}$ 346.0948, found 346.0949 .

4-(8-羊基喹啉-5-亚氨基甲基)-7-己氧基苯并吡喃2-酩(3b): 黄色固体，产率 65\%. m.p. $245 \sim 248{ }^{\circ} \mathrm{C} ;{ }^{1} \mathrm{H}$ NMR (400 MHz, DMSO- $\left.d_{6}\right) \delta: 0.89(\mathrm{t}, J=6.8 \mathrm{~Hz}, 3 \mathrm{H})$, $1.28 \sim 1.35(\mathrm{~m}, 4 \mathrm{H}), 1.37 \sim 1.48(\mathrm{~m}, 2 \mathrm{H}), 1.68 \sim 1.78(\mathrm{~m}$, $2 \mathrm{H}), 4.09(\mathrm{t}, J=6.8 \mathrm{~Hz}, 2 \mathrm{H}), 6.94 \sim 7.05(\mathrm{~m}, 3 \mathrm{H}), 7.56(\mathrm{~d}$, $J=8.4 \mathrm{~Hz}, 1 \mathrm{H}), 7.92(\mathrm{~d}, J=8.4 \mathrm{~Hz}, 1 \mathrm{H}), 8.04$ (dd, $J=5.2$, $8.8 \mathrm{~Hz}, 1 \mathrm{H}), 8.74(\mathrm{~d}, J=8.8 \mathrm{~Hz}, 1 \mathrm{H}), 9.13(\mathrm{~s}, 1 \mathrm{H}, \mathrm{CH}=$ $\mathrm{N}), 9.15(\mathrm{~s}, 1 \mathrm{H}), 9.36(\mathrm{~d}, J=8.8 \mathrm{~Hz}, 1 \mathrm{H}), 10.04(\mathrm{~s}, 1 \mathrm{H}$, $\mathrm{OH}) ;{ }^{13} \mathrm{C}$ NMR (100 MHz, DMSO- $\left.d_{6}\right) \delta: 162.35,160.92$, $160.89,156.23,146.16,142.76,137.62,129.03,128.15$, $127.37,126.41,123.07,115.11,113.68,113.30,113.28$, $110.56,101.99,101.93,68.77,31.39,28.84,25.55,22.52$, 14.38; FT-IR (KBr) v: 3439, 3016, 2928, $1720(\mathrm{C}=\mathrm{O})$, $1615(\mathrm{C}=\mathrm{N}), 1550,1388,1294,1198,1146,1078 \mathrm{~cm}^{-1}$; EI-MS $m / z$ (\%): $416\left([\mathrm{M}]^{+}, 30\right)$. HRMS calcd for $\mathrm{C}_{25} \mathrm{H}_{24} \mathrm{~N}_{2} \mathrm{O}_{4} 416.1731$, found 416.1731 .

4-(8-羊基喹啉-5-亚氨基甲基)-7-十八碳烷氧基苯并 吡喃-2-酮(3c): 黄色固体, 产率 78\%. m.p. 168 170 ${ }^{\circ} \mathrm{C}$; ${ }^{1} \mathrm{H}$ NMR (400 MHz, DMSO- $\left.d_{6}\right) \delta: 0.81(\mathrm{t}, J=6.8 \mathrm{~Hz}$, $3 \mathrm{H}), 1.19 \sim 1.96(\mathrm{~m}, 32 \mathrm{H}), 3.99(\mathrm{t}, J=6.8 \mathrm{~Hz}, 2 \mathrm{H}), 6.70(\mathrm{~s}$, $1 \mathrm{H}), 6.83(\mathrm{~d}, J=2.4 \mathrm{~Hz}, 1 \mathrm{H}), 6.89$ (dd, $J=2.4,9.2 \mathrm{~Hz}$, $1 \mathrm{H}), 7.15$ (d, $J=8.4 \mathrm{~Hz}, 1 \mathrm{H}), 7.18(\mathrm{~d}, J=8.4 \mathrm{~Hz}, 1 \mathrm{H}), 7.29$ 
(d, $J=8.4 \mathrm{~Hz}, 1 \mathrm{H}), 7.50(\mathrm{dd}, J=4.0,8.4 \mathrm{~Hz}, 1 \mathrm{H}), 8.68$ (s, $1 \mathrm{H}), 8.71(\mathrm{~d}, J=2.0 \mathrm{~Hz}, 1 \mathrm{H}), 8.80(\mathrm{dd}, J=1.2,4.0 \mathrm{~Hz}$, 1H), 10.00 (s, 1H); FT-IR (KBr) v: 3449, 2917, 2851, 1695 $(\mathrm{C}=\mathrm{O}), 1618(\mathrm{C}=\mathrm{N}), 1508,1470,1380,1297,1198$, 1141, $1015 \mathrm{~cm}^{-1}$; EI-MS m/z (\%): 584 ([M] $\left.]^{+}, 10\right)$; HRMS calcd for $\mathrm{C}_{37} \mathrm{H}_{48} \mathrm{~N}_{2} \mathrm{O}_{4}$ 584.3609; found 584.3608.

\section{6 金属铝配合物 $\mathrm{Al}(3 \mathrm{a})_{3} \sim \mathrm{Al}(3 \mathrm{c})_{3}$ 的合成}

将 $55.6 \mathrm{mg}(0.16 \mathrm{mmol})$ 配体 $\mathbf{3 a}$ 加入盛有 $10 \mathrm{~mL}$ DMF 的圆底烧瓶中并加热使之完全溶解, 冷却至室温; 再将 $6.8 \mathrm{mg}$ 氯化铝溶于 $5 \mathrm{~mL}$ 甲醇溶液中, 在氮气保护 下，用恒压漏斗将氯化铝甲醇溶液缓慢滴加到盛有 $\mathbf{3 a}$ 的圆底烧瓶中并不断搅拌(约 $30 \mathrm{~min}$ ), 滴加完毕, 升温 到 $80{ }^{\circ} \mathrm{C}$, 继续反应 $12 \mathrm{~h}$, 有褐色沉淀析出, 将反应混合 液静置 $2 \mathrm{~h}$, 离心分离出沉淀, 用少量无水乙醇洗涤 2 3 次, 真空干燥得 $\mathrm{Al}(3 \mathrm{a})_{3}$ 褐色固体 $21 \mathrm{mg}$, 产率 37\%. m.p. $>300{ }^{\circ} \mathrm{C}$; FT-IR (KBr) v: 3351, 1711, 1661, 1605, 1499, 1466, 1388, 1324, 1284, 1208, 1142, $1104 \mathrm{~cm}^{-1}$. Anal. calcd for $\mathrm{C}_{60} \mathrm{H}_{39} \mathrm{~N}_{6} \mathrm{O}_{12} \mathrm{Al}$ : C 67.80, H 3.70, N 7.91; found C 67.08, H 3.86, N 7.29.

$\mathrm{Al}(\mathbf{3 b})_{3}$ 的制备方法与 $\mathrm{Al}(\mathbf{3 a})_{3}$ 相同, 真空干燥得 $\mathrm{Al}(3 \mathrm{~b})_{3}$ 褐色固体, 产率 $40 \%$. m.p. $>300{ }^{\circ} \mathrm{C}$; FT-IR (KBr) v: 3379, 1604, 1495, 1467, 1385, 1322, 1283, 1207, 1141, $1105 \mathrm{~cm}^{-1}$. Anal. calcd for $\mathrm{C}_{75} \mathrm{H}_{69} \mathrm{~N}_{6} \mathrm{O}_{12} \mathrm{Al}$ : C 70.74, $\mathrm{H}$ 5.46, N 6.60; found C 70.24, H 5.76, N 6.51.

$\mathrm{Al}(\mathbf{3 c})_{3}$ 的制备方法与 $\mathrm{Al}(\mathbf{3 a})_{3}$ 相同, 真空干燥得 $\mathrm{Al}(3 \mathrm{c})_{3}$ 褐色固体, 产率 $33.3 \%$. m.p. $>300{ }^{\circ} \mathrm{C}$; FT-IR (KBr) v: 3296, 2923, 1717, 1607, 1503, 1465, 1385, 1264, $1149 \mathrm{~cm}^{-1}$. Anal. calcd for $\mathrm{C}_{111} \mathrm{H}_{141} \mathrm{~N}_{6} \mathrm{O}_{12} \mathrm{Al}$ : C 74.97, $\mathrm{H}$ 7.99, N 4.73; found C 74.62, H 8.05, N 4.52.

\section{7 金属锌配合物 $\mathrm{Zn}(3 \mathrm{a})_{2} \sim \mathrm{Zn}(3 \mathrm{c})_{2}$ 的合成}

将 $41.5 \mathrm{mg}$ (0.12 mmol)配体 $\mathbf{3 a}$ 溶于盛有 $5 \mathrm{~mL} \mathrm{DMF}$ 的圆底烧瓶中并加热使之完全溶解, 冷却至室温, 再将 $11 \mathrm{mg}(0.09 \mathrm{mmol}) 乙$ 酸锌溶于 $5 \mathrm{~mL}$ 无水乙醇溶液中, 在氮气保护下, 用恒压漏斗将乙酸锌乙醇溶液缓慢滴加 到反应瓶中(约 $30 \mathrm{~min}$ ), 滴加完毕, 升温到 $80{ }^{\circ} \mathrm{C}$, 继续 反应 $12 \mathrm{~h}$, 有褐色沉淀析出, 将反应混合液静置 $2 \mathrm{~h}$, 离 心分离出沉淀, 用少量无水乙醇洗涤沉淀 $2 \sim 3$ 次, 真空 干燥得 $\mathrm{Zn}(3 \mathrm{3a})_{2}$ 褐色固体 $22 \mathrm{mg}$, 产率 48\%. m.p. $>300$ ${ }^{\circ} \mathrm{C}$; FT-IR (KBr) v: 3285, 1711, 1613, 1552, 1492, 1465, $1388,1325,1283,1256,1133,512,450 \mathrm{~cm}^{-1}$. Anal. calcd for $\mathrm{C}_{40} \mathrm{H}_{26} \mathrm{~N}_{4} \mathrm{O}_{8} \mathrm{Zn}$ : $\mathrm{C}$ 63.54, $\mathrm{H}$ 3.47, $\mathrm{N}$ 7.41; found $\mathrm{C}$ 63.35, H 3.86, N 7.13.

$\mathrm{Zn}(\mathbf{3 b})_{2}$ 的制备方法与 $\mathrm{Zn}(\mathbf{3 a})_{2}$ 相同, 真空干燥得 $\mathrm{Zn}(3 \mathrm{~b})_{2}$ 褐色固体, 产率 44\%. m.p. $>300{ }^{\circ} \mathrm{C}$; FT-IR (KBr) v: 3076, 2928, 1720, 1610, 1552, 1495, 1462, 1385, 1314,
1286, 1144, 1075, 1006, $502 \mathrm{~cm}^{-1}$. Anal. calcd for $\mathrm{C}_{50} \mathrm{H}_{46} \mathrm{~N}_{4} \mathrm{O}_{8} \mathrm{Zn}$ : C 67.00, H 5.17, N 6.25; found C 67.13, H 5.56, N 7.02 .

$\mathrm{Zn}(\mathbf{3 c})_{2}$ 的制备方法与 $\mathrm{Zn}(\mathbf{3 a})_{2}$ 相同, 真空干燥得 $\mathrm{Zn}(3 \mathrm{c})_{2}$ 褐色固体, 产率 $36 \%$. m.p. $>300{ }^{\circ} \mathrm{C}$; FT-IR $(\mathrm{KBr})$ $v: 3318,2923,2846,1709,1613,1492,1462,1388,1322$, $1292,1196,1146,520 \mathrm{~cm}^{-1}$. Anal. calcd for $\mathrm{C}_{74} \mathrm{H}_{94} \mathrm{~N}_{4^{-}}$ $\mathrm{O}_{8} \mathrm{Zn}$ : C 72.09, H 7.68, N 4.54; found C 72.02, H 7.89, N 4.31 .

\section{2 结果与讨论}

\section{1 波谱及物理性质}

化合物 3a, 3b 和 3c 的红外光谱在 $3400 \sim 3450 \mathrm{~cm}^{-1}$ 均出现 $\mathrm{OH}$ 的伸缩振动吸收峰; 在 $1690 \sim 1720 \mathrm{~cm}^{-1}$ 区 域有 $\mathrm{C}=\mathrm{O}$ 伸缩振动吸收峰; 在 $1620 \sim 1610 \mathrm{~cm}^{-1}$ 区域 有 $\mathrm{C}=\mathrm{N}$ 双键的伸缩振动吸收峰; 在 $1400 \sim 1580 \mathrm{~cm}^{-1}$ 区域均有苯环的骨架的伸缩振动吸收峰; 在 1000 $1200 \mathrm{~cm}^{-1}$ 之间有 $\mathrm{C}-\mathrm{O}$ 键的伸缩振动吸收峰. 在 ${ }^{1} \mathrm{H}$ NMR 中, 化合物 $3 \mathbf{a}$ 和 $\mathbf{3 b}$ 在 $\delta$ 为 9.13 和 9.15 处均出现 一个 $\mathrm{H}$ 氢的单峰, 可确定该氢为 $\mathrm{N}=\mathrm{CH}$ 上的氢信号; 另外 EI-MS 数据, 可见到 $m / z$ 346, 416 和 584 的分子离 子峰; HRMS 数据确定的化合物分子式与 $\mathbf{3 a}, \mathbf{3 b}$ 和 $\mathbf{3 c}$ 一 致. 这些数据表征了化合物的结构.

金属配合物 $\mathrm{Al}(\mathbf{3 a})_{3} \sim \mathrm{Al}(\mathbf{3 c})_{3}, \mathrm{Zn}(\mathbf{3 a})_{2} \sim \mathrm{Zn}(\mathbf{3} \mathbf{c})_{2}$ 的红 外光谱数据比较发现, 配体 3a, 3b 和 3c 中羟基 3400 $3450 \mathrm{~cm}^{-1}$ 出现的 $\mathrm{OH}$ 伸缩振动峰在形成铝、锌配合物 以后波数降低到 $3400 \sim 3100 \mathrm{~cm}^{-1}$ 范围; 与金属铝配位 后, 在 $1100 \mathrm{~cm}^{-1}$ 附近出现了明显的特征吸收峰, 为 $\mathrm{Al}-\mathrm{O}-\mathrm{C}$ 中的 $\mathrm{O}-\mathrm{C}$ 键伸缩振动所致 ${ }^{[17]}$; 另外锌配合 物在 $520 \sim 480 \mathrm{~cm}^{-1}$ 范围出现新的 $\mathrm{Zn}-\mathrm{N}$ 弱吸收峰, 在 $420 \sim 450 \mathrm{~cm}^{-1}$ 范围出现新的 $\mathrm{Zn}-\mathrm{O}$ 弱吸收峰 ${ }^{[8]}$, 说明 $\mathrm{Al}^{3+}, \mathrm{Zn}^{2+}$ 参与了配位.

香豆素和 8-差基喹啉可溶于相应的溶剂中, 二者缩 合成席夫碱后, 其溶解性能降低, 为了使席夫碱配体能 保持一定的溶解性，我们对香豆素 7-位上的羟基进行了 不同程度的烷基化修饰，结果显示这种修饰作用对 3a 3c 配体及其铝、锌配合物的溶解性能并未产生大的 影响.

\section{2 配体及金属配合物的紫外吸收光谱}

配体及金属配合物试样浓度均为 $1 \times 10^{-5} \mathrm{~mol} / \mathrm{L}$, 溶剂为 DMSO, 吸收光谱如图 1A $1 \mathrm{C}$ 所示, 8-差基喹啉 与香豆素形成的席夫碱衍生物配体 $\mathbf{3 a}, \mathbf{3 b}$ 和 $\mathbf{3 c}$ 均在 247 $\mathrm{nm}$ 附近有较大的吸收, 而 $\mathbf{3 a}$ 和 $\mathbf{3 b}$ 分别还在 322 和 324 $\mathrm{nm}$ 处出现最大吸收峰, $\mathbf{3 c}$ 在此处的吸收减弱, 仅在 325 $\mathrm{nm}$ 仅有一微弱的吸收. $\mathbf{3 a}, \mathbf{3 b}$ 和 $\mathbf{3 c}$ 的铝配合物及 $\mathbf{3 c}$ 的 
锌配合物均在 $332 \mathrm{~nm}$ 处有最大吸收, $\mathbf{3 a}$ 和 $\mathbf{3 b}$ 的锌配合 物在 $328 \mathrm{~nm}$ 处有最大吸收, 最大吸收的位置比相应配 体红移 4 10 nm, 这符合配合物分子中共轭程度增大 会导致吸收光谱红移的规律, 也表明配体中的 $\pi-\pi$ *跃迁 在金属配合物中依然保持, 但其 $\varepsilon$ 值明显降低, 其中铝 配合物的 $\varepsilon$ 值随着侧链的增长有所降低, 而锌配合物的
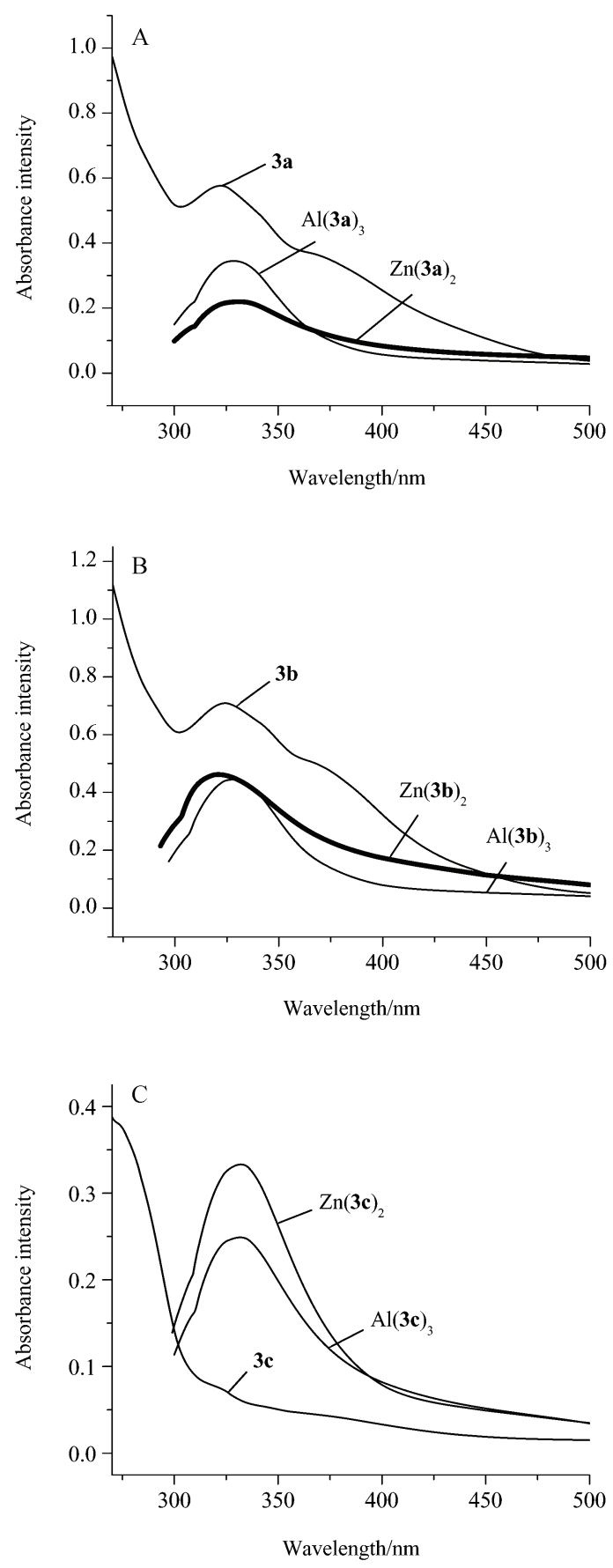

图 1 三种配体及其金属配合物的紫外光谱图

Figure 1 The UV absorption spectra of three ligands and their metal complexes

$\mathrm{A}, \mathrm{B}$ and $\mathrm{C}$ stand for the UV absorption spectra of $\mathbf{3 a}, \mathbf{3} \mathbf{b}$ and $\mathbf{3} \mathbf{c}$ and their metal complexes, respectively $\varepsilon$ 值则随着侧链的增长有所升高.

\section{3 配体及金属配合物的荧光光谱}

配体及金属配合物苂光测试试样浓度均为 $1 \times 10^{-5}$ $\mathrm{mol} / \mathrm{L}$, 溶剂为 $\mathrm{DMSO}$, 配体 $\mathbf{3 a} \sim \mathbf{3} \mathbf{c}$ 以及其金属 $\mathrm{Al}$ 和 $\mathrm{Zn}$ 配合物的荧光发射光谱都选择最大激发波长作为激 发光波长, 如图 $2 \mathrm{~A} \sim 2 \mathrm{C}$ 所示.

配体 3a 3c 的荧光光谱最大发射波长在 $450 \sim 458$ $\mathrm{nm}$ 之间; $\mathrm{Al}(\mathbf{3 a})_{3} \sim \mathrm{Al}(3 \mathbf{c})_{3}$ 的荧光发射波长分别为 462 , 465 和 $440 \mathrm{~nm} ; \mathrm{Zn}(3 \mathbf{a})_{2} \sim \mathrm{Zn}(3 \mathbf{c})_{2}$ 的荧光发射波长分别 为 560,472 和 $461 \mathrm{~nm}$.

图 2A 是 3a 及其金属配合物在 DMSO 中的苂光发 射光谱，化合物 $\mathbf{3 a}$ 在最大激发波长 $322 \mathrm{~nm}$ 的光激发下， $3 \mathbf{a}$ 仅在 $450 \mathrm{~nm}$ 处出现一个弱的苂光发射峰，表明配体 本身能发出较弱的荧光，而对其金属配合物 $\mathrm{Al}(\mathbf{3 a})_{3}$ 在 激发波长 $332 \mathrm{~nm}$ 的光激发下，在 $450 \sim 600 \mathrm{~nm}$ 出现一个 稍大的荧光发射峰，峰值在 $462 \mathrm{~nm}$ 处，发蓝色荧光; 对 于金属配合物 $\mathrm{Zn}(\mathbf{3 a})_{2}$ 在激发波长 $328 \mathrm{~nm}$ 的光激发下, 在 $525 \sim 650 \mathrm{~nm}$ 出现一个较强的荧光发射峰, 峰值在 $560 \mathrm{~nm}$ 处, 介于绿光和橙红光之间，说明此种配体的锌 配合物比铝配合物有较强的苂光发射调控能力. 这种配 合物苂光的产生属于金属离子微扰配体发光.

图 2B 是 $3 \mathbf{b}$ 及其金属配合物在 DMSO 中的荧光发 射光谱. 化合物 $\mathbf{3 b}$ 在最大激发波长 $324 \mathrm{~nm}$ 的光激发下, 3b 仅在 $458 \mathrm{~nm}$ 处出现一个弱的荧光发射峰, 表明配体 本身能发出较弱的苂光，而对其金属配合物 $\mathrm{Al}(3 \mathbf{b})_{3}$ 在 激发波长 $332 \mathrm{~nm}$ 的光激发下，在 $400 \sim 600 \mathrm{~nm}$ 出现一个 稍强的荧光发射峰，峰值在 $465 \mathrm{~nm}$ 处，发蓝色荧光; 对 于金属配合物 $\mathrm{Zn}(3 \mathbf{b})_{2}$ 在激发波长 $328 \mathrm{~nm}$ 的激发下，在 $400 \sim 650 \mathrm{~nm}$ 出现一个较强的苂光发射峰, 峰值在 472 $\mathrm{nm}$ 处，发蓝色苂光. $3 \mathbf{b}$ 配合物荧光的产生与配体 $\mathbf{3 a}$ 一 样也属于金属离子微扰配体发光，可以使用此方法获得 复合发光材料.

图 2C 是 3c 及其金属配合物在 DMSO 中的苂光发 射光谱. 化合物 $3 \mathbf{c}$ 在最大激发波长 $325 \mathrm{~nm}$ 的光激发下, 3c 仅在 457 处出现一个稍强的荧光发射峰, 表明配体本 身能发出较弱的荧光, 而对于金属配合物 $\mathrm{Al}(3 \mathbf{c})_{3}$ 在其 激发波长 $332 \mathrm{~nm}$ 的光激发下，在 $400 \sim 600 \mathrm{~nm}$ 出现一个 很强的菼光发射峰，峰值在 $440 \mathrm{~nm}$ 处，发蓝色苂光; 对 于金属配合物 $\mathrm{Zn}(\mathbf{3 c})_{2}$ 在激发波长 $332 \mathrm{~nm}$ 的光激发下, 在 $400 \sim 650 \mathrm{~nm}$ 出现一个弱的苂光发射峰，峰值在 520 $\mathrm{nm}$ 处，介于蓝光和绿光之间. 这种配合物荧光的产生 也同属于金属离子微扰配体发光.

从 3a, 3b 和 3c 在 DMSO 中的荧光发射光谱来看, 香豆素 7-位的烷氧基侧链对配体荧光发射波长影响不 

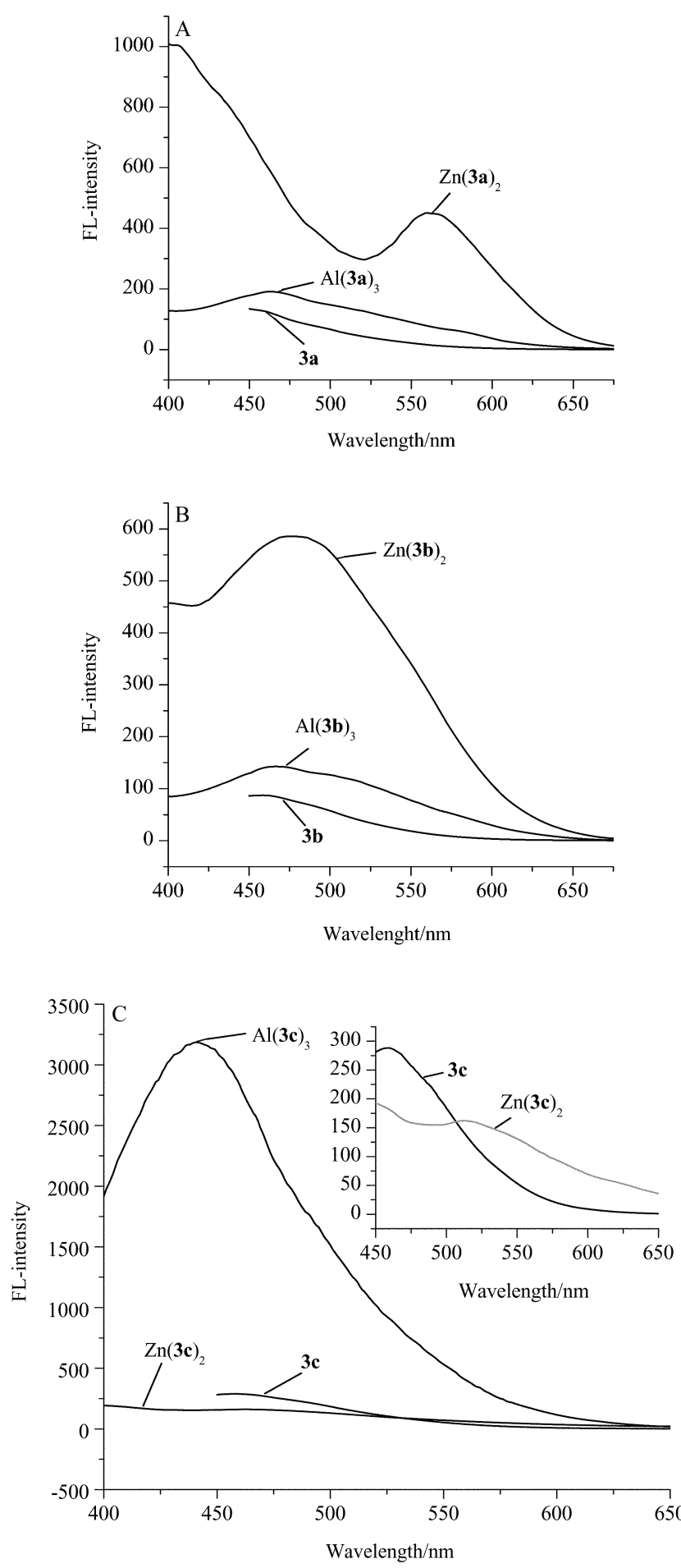

图 2 三种配体及其金属配合物的苂光发射光谱图

Figure 2 The fluorescence emission spectra of three ligands and their metal complexes

$\mathrm{A}, \mathrm{B}$ and $\mathrm{C}$ stand for the fluorescence emission spectra of $\mathbf{3 a}, \mathbf{3 b}$ and $\mathbf{3 c}$ and their metal complexes, respectively

大，最大激发波长在 $450 \sim 458 \mathrm{~nm}$ 之间，都在蓝光发光 区域内. 从 $3 \mathrm{a}, 3 \mathrm{~b}$ 和 $3 \mathrm{c}$ 的金属铝配合物在 DMSO 中的
荧光发射光谱发现，香豆素 7-位的烷氧基侧链在六碳以 内对其发射波长和强度影响不大, 如 $\mathrm{Al}(\mathbf{3 a})_{3}$ 和 $\mathrm{Al}(\mathbf{3 b})_{3}$ 的发射波长分别是 462 和 $465 \mathrm{~nm}$, 在十八碳烷氧基取代 时发射波长向蓝移 $22 \sim 25 \mathrm{~nm}$ ，且强度显著增强，但都 在蓝光发光区域内. 从 $3 \mathrm{a}, 3 \mathrm{~b}$ 和 $3 \mathrm{c}$ 的金属锌配合物在 DMSO 中的苂光发射光谱发现，香豆素 7-位为甲氧基侧 链时，最大荧光发射波长是 $560 \mathrm{~nm}$ ，发绿色光，而 $3 \mathbf{b}$ 和 $3 \mathrm{c}$ 的最大荧光发射波长分别是 472 和 $461 \mathrm{~nm}$, 发蓝色 光. 产生这种差别的原因是可能是甲氧基的供电子的能 力较强，基态电子更容易跃迁，因此发生明显的红移， 而随着烷基链的长度增长, 烷氧基的供电子的能力减 弱，仍保持在蓝色发光区域. 另外由于金属铝是形成三 配体的配合物，而金属锌是形成二配体的配合物，金属 铝配合物的拥挤程度要比锌配合物大，因此金属铝配合 物受到香豆素侧链烷氧基的影响要比金属锌配合物小.

\section{References}

[1] Tang, C. W.; Vanslyke, S. A. Appl. Phys. Lett. 1987, 51, 913.

[2] Burrows, H. D.; Fernandes, M.; Seixas de Melo, J.; Monkman, A. P.; Navaratnam, S. J. Am. Chem. Soc. 2003, 125, 15310.

[3] Hosokawa, C.; Tokailin, H.; Higashi, H.; Kusumoto, T. Appl. Phys. Lett. 1992, 60, 1220.

[4] Matsumura, M.; Akai, T. Jpn. J. Appl. Phys. 1996, 35, 5357.

[5] Garbuzov, D. Z.; Bulovic, V.; Burrows, P. E.; Förrest, S. R. Chem. Phys. Lett. 1996, 249, 433.

[6] Burrows, P. E.; Shen, Z.; Bulovic, V.; McCarty, D. M.; Förrest, S T.; Cronin, J. A.; Thompson, M. E. J. Appl. Phys. 1996, 79, 7991.

[7] Chen, C.-H.; Shi, J. M. Coord. Chem. Rev. 1998, 171, 161.

[8] Ouyang, X.-H.; Zeng, H.-P.; Xie, Y. Chin. J. Org. Chem. 2007, 27, 402 (in Chinese) (欧阳新华，曾和平，谢彦，有机化学， 2007, 27, 402.)

[9] Pohl, R.; Anzenbacher, P. Jr. Org. Lett. 2003, 5, 2769.

[10] Pohl, R.; Montes, V. A.; Shinar, J.; Anzenbacher, P. Jr. J. Org. Chem. 2004, 69, 1723.

[11] Burrows, P. E.; Sapochak, L. S.; McCarty, D. M.; Förrest, S. R.; Thompson, M. E. Appl. Phys. Lett. 1994, 64, 2718.

[12] Tang, C. W.; Vanslyke, S. A.; Chen, C.-H. J. Appl. Phys. 1989, 65, 3610.

[13] Lee, M. T.; Yen, C. K.; Yang, W.-P.; Chen, H.-H.; Liao, C.-H.; Tsai, C. H.; Chen, C.-H. Org. Lett. 2004, 6, 1241.

[14] Swanson, S. A.; Wallraff, G. M.; Chen, J.-P.; Zhang, W.-J.; Bozano, L. D.; Carter, K. R.; Salem, J. R.; Villa, R.; Scott, J. C. Chem. Mater. 2003, 15, 2305.

[15] Alexander, V. M.; Bhat, R. P.; Samant, S. D. Tetrahedron Lett. 2005, 46, 6957.

[16] Xu, S.; Xu, S.-P.; Li, L.-M. Acta Pharm. Sinica 2001, 36, 269 (in Chinese).

(徐嵩, 徐世平, 李兰敏, 药学学报, 2001, 36, 269.)

[17] Fan, L.-Q.; Zhu, W.-H.; Li, J.; Yang, S.-J.; Tian, H. Chemistry 2004, 67, 50 (in Chinese).

(范立强, 朱为宏, 李晶, 杨松杰, 田禾, 化学通报, 2004, 67, 50.) 